\title{
Hawking process in solids: Quantum generation of phonon bursts by a strongly excited mode
}

\author{
Vladimir Hizhnyakov and Dmitri Nevedrov \\ Institute of Theoretical Physics, University of Tartu, \\ Tähe 4, EE2400 Tartu, Estonia. \\ Institute of Physics, Riia 142, EE2400 Tartu, Estonia.
}

\begin{abstract}
We examine quantum decay of localized vibrations in anharmonic crystal lattice. The theory which describes two-phonon anharmonic relaxation can be applied both to local modes associated with substitutional impurity and to intrinsic local modes (ILM) in perfect lattices. It is found that for sufficiently high initial excitations relaxation of vibrations is non-exponential, it demonstrates explosion-like behavior at specific stages of evolution. The course of the relaxation is determined by the initial value of energy, temperature, direction of vibrations. As an example we present the results of calculations of the relaxation of an odd local (impurity) mode in a simple cubic lattice and discuss the influence of quantum fluctuations on the stability of the ILM in one-dimensional monatomic chain.
\end{abstract}

\section{INTRODUCTION}

The localization and energy transport in nonlinear systems have received much attention recently. Numerical techniques play an essential role in the investigation of anharmonic discrete lattices, allowing the variation of model parameters and the observation of different properties. The existence of stationary localized vibrations in a perfect chain $[1,2,3,4,5]$ is of particular interest as it links local lattice dynamics with physics of solitons.

So far the research of strong anharmonic effects in lattice dynamics as well as molecular dynamics simulations of the kinetics of the decay of vibrational excitations [6] has been carried out within the frame of classical mechanics. However, the account of quantum effects may be important, as it leads to creation of new channels in the decay. In this context the problem of strong local vibration is also of significant interest, it gives an example when anharmonic and quantum effects can be described analitycally with the application of nonperturbative theory. One of the results of the corresponding theory $[8,9,10]$ is an explosion-like release of energy by the local vibration with the emission of a burst of phonons. This release takes place if the energy of the local mode approaches some definite critical values. Such a behaviour is new for vibrational systems and it is a consequence of a mutual interplay between anharmonic and quantum effects.

The method used in $[8,10]$ is based on the assumption that a local mode is strongly excited initially and it can be considered classically. Phonons are supposed to be not excited (or to be in thermal equilibrum). According to classical mechanics, the state with non-excited phonons is stable, since for the phonon coordinates $q_{i}$ at rest $\left(q_{i}=0\right)$ the anharmonic interaction is turned off. Quantum fluctuations, coming from zero-point vibrations, turn on the interaction and result the relaxation of the mode. We must note, however, that the standard quantum theory, based on the description of anharmonic interaction as a small perturbation [1], cannot be used for the obtaining of the solution to the problem of relaxation, because the interaction is not weak (due to the strong excitation of the local mode). 


\section{GENERAL THEORY}

Let us consider a local vibration anharmonically interacting with phonons. The peculiarity of the problem is that the strong local vibration causes periodic time-dependence of the local force constants and therefore of the zero-point energy of the phonon system. This time-dependence results the generation of phonons. The mechanism of this process has an analogy with the black hole emission mechanism proposed by Hawking [11] and with Unruh radiation [14]. In all these cases the time dependence of the zero-point energy causes the transformation of the initial creation and destruction operators in time $[12,13]$. Namely, according to Hawking [11]

$$
\hat{b}_{i}=\sum_{i}\left(\bar{\alpha}_{i j} \hat{a}_{j}-\bar{\beta}_{i j} \hat{a}_{j}^{+}\right) \text {, }
$$

where $\hat{a}$ and $\hat{b}$ are initial (incoming) and final (outgoing) time-dependent destruction operators for photons. It means that when there are no incoming particles the number operator of the $i$-th outgoing state is

$$
N_{i}=<0\left|\hat{b}_{i}^{+} \hat{b}_{i}\right| 0>=\sum_{j}\left|\beta_{i j}\right|^{2},
$$

i.e. the number of particles created and emitted in a gravitational collapse can be determined calculating the coefficients $\beta_{i j}$. There exist several situations in quantum field theory where the phenomena similar to black hole evaporation appear. The most famous are:

- Pair production in a static electric field revealed by Heisenberg [21]. Vacuum of quantum field theory is unstable against the creation of charged pairs.

- Accelerated systems which become spontaneously excited in Minkowski - space and accelerated mirrors.

It is also known that the rate of emission of the black hole is very high near the end of its life. As a black hole emits radiation it loses mass. This in turn increase the rate of emission. Near the end of its life about $10^{30} \mathrm{erg}$ would be released in $0.1 \mathrm{~s}$. So the emitting rate of the black hole grows in time and at some critical moment a large amount of energy will be released in very short time (explosion). We will point out how the analogy of this dramatic effect appears in the system of phonons in crystal.

Cubic anharmonic interaction of the local mode with crystalline is the sum of terms $\sim \hat{x}_{i} \hat{x}_{i^{\prime}} Q(t)$, where $\hat{x}_{i}$ and $\hat{x}_{i^{\prime}}$ are the operators of normal coordinates of crystalline phonons, $Q(t)$ is the timedependent classical amplitude of the local mode. This interaction leads to the following timedependent Hamiltonian of the phonon system:

$$
\hat{H}_{p h}(t)=\frac{1}{2} \sum_{i}\left(\dot{\hat{x}}_{i}^{2}+\omega_{i}^{2} \hat{x}_{i}^{2}\right)+\frac{1}{2} Q(t) \sum_{i i^{\prime}}\left(e_{i} w e_{i^{\prime}}\right) \hat{x}_{i} \hat{x}_{i^{\prime}}
$$

Here $\omega_{i}$ is the frequency of the mode $i,\left(e_{i} w e_{i^{\prime}}\right) \equiv \sum_{n} e_{i n} e_{i^{\prime} n} w_{n n}, n=1,2, \ldots, n_{0}, \sum_{i} e_{i n} e_{i n^{\prime}}=\delta_{n n^{\prime}}, n_{0}$ is the number of configurational coordinates contributing to anharmonic interaction.

The Hamiltonian (3) can be diagonalized as follows [8]:

$$
\hat{H}_{p h}(t)=\sum_{j} \hbar \Omega_{j}(t)\left(\hat{b}_{j}^{+}(t) \hat{b}_{j}(t)+\frac{1}{2}\right)
$$

where $\Omega_{j}(t)$ are time-dependent phonon frequencies, and

$$
\hat{b}_{j}(t)=\sum_{i}\left(\mu_{i j}(t) \hat{a}_{i}+\nu_{i j}(t) \hat{a}_{i}^{+}\right)
$$

are time-dependent destruction operators; expressions for $\Omega_{j}, \mu_{i j}$ and $\nu_{i j}$ are given in [19].

The anharmonic interaction considered causes not only time dependence of phonon frequencies but also changes of phonon operators in time. Note that relation (5) is analogous to the relation of Hawking (1) between field operators in different times of gravitationally collapsing star. This is why the mechanism of a local mode relaxation is analogous to that of black hole emission: phonons 
(photons) are generated because the initial zero-point state $\mid 0>$ is not zeroth state for the timedependent destruction operators $\hat{b}_{j}$; there are phonons with frequencies $\Omega_{j}$ in $\mid 0>$ at the time moment $t$; the number of phonons equals $\sum_{j} N_{j}(t)$, where $N_{j}(t)=\left\langle 0\left|\hat{b}_{j}^{+}(t) \hat{b}_{j}(t)\right| 0\right\rangle$.

Energy, which is generated in phonon system at the time moment $t$, equals $[8,9]$ :

$$
E_{p h}(t)=\sum_{j} \hbar \Omega_{j}(t)\left(N_{j}(t)+\frac{1}{2}\right)-\sum_{i} \frac{\hbar \omega_{i}}{2}=\frac{\hbar}{4} \sum_{i i^{\prime}} \omega_{i}^{-1} e_{i}^{2} e_{i^{\prime}}^{2}\left|\int_{0}^{t} D_{i}(\tau) e^{-i \omega_{i^{\prime}} \tau} d \tau\right|^{2},
$$

(fast oscillating terms are neglected). This energy comes from the local mode: $E_{p h}(t)=E_{l}(0)-E_{l}(t)$; $E_{l}(t) \simeq \omega_{l}^{2} Q_{0}^{2}(t) / 2, Q_{0}(t)$ is the mode amplitude, $\omega_{l}$ is its frequency. This relation between the energies $E_{p h}$ and $E_{l}$ gives an equation for $E_{l}(t)$. Differentiating (6) and taking into account that $d E_{p h}=-d E_{l}$, one obtains $\dot{E}_{l}(t) \approx-\gamma(t) E_{l}(t)$, where

$$
\gamma(t) \approx \frac{\pi \hbar w^{2}}{4 \omega_{l}} \sum_{k=1}^{m+1} \int_{\bar{\omega}_{k-1}}^{\bar{\omega}_{k}} \frac{d \omega \rho(\omega) \rho\left(\omega_{l}-\omega\right)}{\left|1-w^{2} E_{l}(t) \tilde{G}\left(\omega-\omega_{l}\right) / 2 \omega_{l}^{2}\right|^{2}}\left[1-\Theta\left(E_{k}-E_{l}(t)\right) e^{-2 \Gamma_{k} t}\right]
$$

stands for the relaxation rate at the time moment $t\left(\bar{\omega}_{0}=0, \bar{\omega}_{m+1}=\omega_{l} / 2\right), \rho(\omega)=-\Im(G(\omega))$ is the phonon density of states (for $\omega>0), \tilde{G}\left(\omega-\omega_{l}\right)=G(\omega) G\left(\omega-\omega_{l}\right)$ is the two-phonon Green's function, expression for $\Gamma_{k}$ is given in $[9,10]$. The second term in square brackets in $(7)$ is important only if the initial energy is close to critical point. When deriving expression (7) it was taken into account that for $E_{l} \approx E_{k}$

$$
\gamma_{k} \sim \frac{1-\Theta\left(E_{k}-E_{l}(t)\right) e^{-2 \Gamma_{k} t}}{E_{k}-E_{l}(t)} .
$$

\section{ODD LOCAL MODE IN A CUBIC LATTICE}

To illustrate the theory we calculate the relaxation of the local mode associated with a light substitutional impurity atom in a simple cubic lattice. Within the approximation of the nearest neighbours interaction the potential operator has the form

$$
\hat{V}=\sum_{\alpha} \sum_{\vec{n}} \sum_{m=1}^{\infty} \frac{1}{m} K_{\bar{n}_{\alpha}}^{(m)}\left(\hat{R}_{\vec{n}_{\alpha}}\right)^{m},
$$

where $\alpha=x, y, z$ are the directions of crystal axes, $\vec{n}=\left(n_{x}, n_{y}, n_{z}\right)$ is the vector of the lattice sites, $\vec{n}_{\alpha}$ is the vector of the site nearest to $\vec{n}$ in $\alpha$ direction,

$$
\hat{R}_{\vec{n}_{\alpha}}=\sqrt{\left(R_{0 n_{\alpha}}+\hat{r}_{\alpha \vec{n}_{\alpha}}\right)^{2}+\hat{r}_{\vec{n}_{\alpha}}^{2}-\hat{r}_{\alpha \vec{n}_{\alpha}}^{2}}
$$

is the operator of distance between the nearest neighbours in the $\alpha$-direction, $R_{0 \vec{n}_{\alpha}}$ is the distance between the nearest neigbour sites, $\hat{r}_{\beta \vec{n}_{\alpha}}=q_{\beta \vec{n}}-q_{\beta \vec{n}_{\alpha}}, q_{\beta}$ is the $\beta$-component of the displacement vector $\vec{q}_{\vec{n}}$ of the atom $\vec{n}, \hat{r}_{\vec{n}_{\alpha}}^{2}=\hat{r}_{x \vec{n}_{\alpha}}^{2}+\hat{r}_{y \vec{n}_{\alpha}}^{2}+\hat{r}_{z \vec{n}_{\alpha}}^{2}$. By expanding $\hat{V}$ in the power series of displacement operators one gets

$$
\hat{V}=\frac{1}{2} \sum_{\alpha, \vec{n}}\left(V_{2 \vec{n}_{\alpha}} \hat{r}_{\vec{n}_{\alpha}}^{2}+V_{2 \vec{n}_{\alpha}}^{\prime} \hat{r}_{\alpha \vec{n}_{\alpha}}^{2}+V_{3 \vec{n}_{\alpha}} \hat{r}_{\bar{n}_{\alpha}}^{2} \hat{r}_{\alpha \vec{n}_{\alpha}}+V_{3 \bar{n}_{\alpha}}^{\prime} \hat{r}_{\alpha \vec{n}_{\alpha}}^{3}\right)+\ldots
$$

where

$$
\begin{aligned}
V_{2 \vec{n}_{\alpha}} & =\sum_{m} R_{0 \bar{n}_{\alpha}}^{m-2} K_{\bar{n}_{\alpha}}^{(m)}, \\
V_{2 \bar{n}_{\alpha}}^{\prime} & =\sum_{m} R_{0 \bar{n}_{\alpha}}^{m-2} K_{\bar{n}_{\alpha}}^{(m)}(m-2), \\
V_{3 \bar{n}_{\alpha}} & =\sum_{m} R_{0 \bar{n}_{\alpha}}^{m-3} K_{\bar{n}_{\alpha}}^{(m)}(m-2), \\
V_{3 \bar{n}_{\alpha}}^{\prime} & =\frac{1}{3} \sum_{m} R_{0 \vec{n}_{\alpha}}^{m-3} K_{\bar{n}_{\alpha}}^{(m)}(m-2)(m-4) .
\end{aligned}
$$




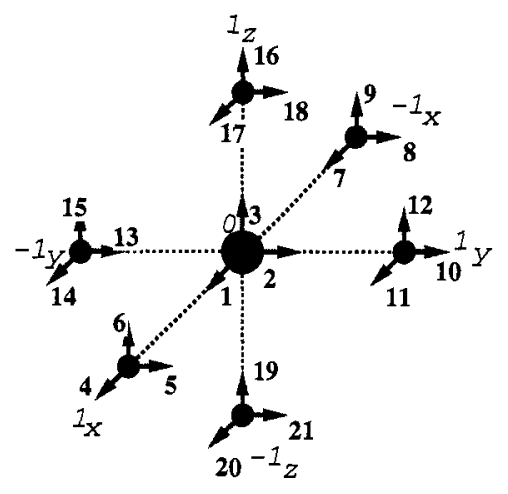

Fig. 1. Displacements which contribute to the relaxation of the central atom. The central (impurity) atom and its six nearest neighbours are shown.

The potential considered does not take account of the covalent interaction which leads to the chemical bonding. This (covalent) interaction can, however, be easily included in calculations by introducing additional terms of the type $V_{2 \vec{n}_{\alpha}}^{\prime}$ and $V_{3 \vec{n}_{\alpha}}^{\prime}$.

We make calculations for a local mode associated with a vibration of a light substitutional impurity atom situated at the origin of our reference frame. In this case the solutions of classical equations of motion, corresponding to the local mode, satisfy the conditions for the mode localized on the impurity atom at the site $n=0$. As a consequence, all mathematical calculations are simplified, since one can neglect contribitions of other atoms to the classical motion. This allows us to neglect also the variations of the frequency of the local mode and of the constants of anharmonic interaction which are caused by the variation of the amplitude of the local mode.

Altogether there are 21 operators of Cartesian coordinates (three for each of 7 atoms includung the central atom and 6 its nearest neighbours) which contribute to the relaxation of the local mode in this approximation. Therefore, the interaction Hamiltonian is

$$
\hat{H}^{\prime}=Q_{l} \cos \omega_{l} t \sum_{n, n^{\prime}=1}^{21} V_{3 n n^{\prime}} \hat{q}_{n} \hat{q}_{n^{\prime}}
$$

The matrix $\left\{V_{3 n n^{\prime}}\right\}$ is determined by the coefficients of the quadratic operators of cubic anharmonicity in (11) with

$$
\hat{r}_{\beta \vec{n}_{\alpha}}=C_{\beta}+\hat{q}_{\beta \vec{n}}-\hat{q}_{\beta \vec{n}_{\alpha}},
$$

where $C_{\beta}$ are Cartesian amplitudes of the local mode; $n$ denotes the numbers of the central atom and its nearest neighbours and the Cartesian components of their coordinates. We choose (see Fig. 1): $\hat{q}_{1}=\hat{x}_{0}, \hat{q}_{2}=\hat{y}_{0}, \hat{q}_{3}=\hat{z}_{0}, \hat{q}_{4}=\hat{x}_{1_{x}}, \hat{q}_{5}=\hat{y}_{1_{x}}, \hat{q}_{6}=\hat{z}_{1_{x}}, \hat{q}_{7}=\hat{x}_{-1_{x}}, \hat{q}_{8}=\hat{y}_{-1_{x}}, \hat{q}_{9}=\hat{z}_{-1_{x}}, \hat{q}_{10}=\hat{y}_{1_{y}}$, $\hat{q}_{11}=\hat{x}_{1_{y}}, \hat{q}_{12}=\hat{z}_{1_{y}}, \hat{q}_{13}=\hat{y}_{-1_{y}}, \hat{q}_{14}=\hat{x}_{-1_{y}}, \hat{q}_{15}=\hat{z}_{-1_{y}}, \hat{q}_{16}=\hat{z}_{1_{z}}, \hat{q}_{17}=\hat{x}_{1_{z}}, \hat{q}_{18}=\hat{y}_{1_{z}}, \hat{q}_{19}=\hat{z}_{-1_{z}}$, $\hat{q}_{20}=\hat{x}_{-1_{z}}, \hat{q}_{21}=\hat{y}_{-1_{z}} ; C=\sqrt{C_{x}^{2}+C_{y}^{2}+C_{z}^{2}}$.

The matrix $w=\left\{V_{3 n n^{\prime}}\right\} / V_{30}$ for three directions in the crystal lattice is given below $\left(V_{30} \equiv V_{30_{\alpha}}\right.$; $\left.\kappa=1+V_{30_{\alpha}}^{\prime} N_{30}\right)$.

The operator $H^{\prime}$ with the given matrices $w$ can be diagonalized analitycally. Since the corresponding final analitycal expressions for the eigenvalues are complicated, we restrict ourselves in giving their non-zero values for some chosen parameters $\kappa$ (see Table 1 and Table 2).

$$
w^{(1)}=\left(\begin{array}{ccccccc}
0 & -M_{1} & M_{1} & -M_{2} & M_{2} & -M_{3} & M_{3} \\
-M_{1} & M_{1} & 0 & 0 & 0 & 0 & 0 \\
M_{1} & 0 & -M_{1} & 0 & 0 & 0 & 0 \\
-M_{2}^{\top} & 0 & 0 & M_{4} & 0 & 0 & 0 \\
M_{2}^{\top} & 0 & 0 & 0 & -M_{4} & 0 & 0 \\
-M_{3}^{\top} & 0 & 0 & 0 & 0 & M_{4} & 0 \\
M_{3}^{\top} & 0 & 0 & 0 & 0 & 0 & -M_{4}
\end{array}\right), \quad[100]
$$




$$
\begin{aligned}
& w^{(2)}=\frac{1}{\sqrt{2}}\left(\begin{array}{ccccccc}
0 & -M_{5} & M_{5} & -M_{6} & M_{6} & -M_{7} & M_{7} \\
-M_{1} & M_{5} & 0 & 0 & 0 & 0 & 0 \\
M_{1} & 0 & -M_{5} & 0 & 0 & 0 & 0 \\
-M_{6}^{\top} & 0 & 0 & M_{5} & 0 & 0 & 0 \\
M_{6}^{\top} & 0 & 0 & 0 & -M_{5} & 0 & 0 \\
-M_{7}^{\top} & 0 & 0 & 0 & 0 & M_{8} & 0 \\
M_{7}^{\top} & 0 & 0 & 0 & 0 & 0 & -M_{8}
\end{array}\right),[\mathbf{1 1 0}], \\
& w^{(3)}=\frac{1}{\sqrt{3}}\left(\begin{array}{ccccccc}
0 & -M_{9} & M_{9} & -M_{10} & M_{10} & -M_{11} & M_{11} \\
-M_{9} & M_{9} & 0 & 0 & 0 & 0 & 0 \\
M_{9} & 0 & -M_{9} & 0 & 0 & 0 & 0 \\
-M_{10}^{\top} & 0 & 0 & M_{9} & 0 & 0 & 0 \\
M_{10}^{\top} & 0 & 0 & 0 & -M_{9} & 0 & 0 \\
-M_{11}^{\top} & 0 & 0 & 0 & 0 & M_{9} & 0 \\
M_{11}^{\top} & 0 & 0 & 0 & 0 & 0 & -M_{9}
\end{array}\right), \quad[111] ; \\
& M_{1}=\left(\begin{array}{lll}
\kappa & 0 & 0 \\
0 & 1 & 0 \\
0 & 0 & 1
\end{array}\right), M_{2}=\left(\begin{array}{lll}
1 & 0 & 0 \\
0 & 1 & 0 \\
0 & 0 & 0
\end{array}\right), M_{3}=\left(\begin{array}{lll}
1 & 0 & 0 \\
0 & 0 & 0 \\
0 & 1 & 0
\end{array}\right), M_{4}=\left(\begin{array}{lll}
0 & 1 & 0 \\
1 & 0 & 0 \\
0 & 0 & 0
\end{array}\right), \\
& M_{5}=\left(\begin{array}{lll}
\kappa & 1 & 0 \\
1 & 1 & 0 \\
0 & 0 & 1
\end{array}\right), M_{6}=\left(\begin{array}{lll}
1 & 1 & 0 \\
\kappa & 1 & 0 \\
0 & 0 & 1
\end{array}\right), M_{7}=\left(\begin{array}{lll}
1 & 0 & 0 \\
1 & 0 & 0 \\
0 & 1 & 1
\end{array}\right), M_{8}=\left(\begin{array}{lll}
0 & 1 & 1 \\
1 & 0 & 0 \\
1 & 0 & 0
\end{array}\right) \text {, } \\
& M_{9}=\left(\begin{array}{lll}
\kappa & 1 & 1 \\
1 & 1 & 0 \\
1 & 0 & 1
\end{array}\right), M_{10}=\left(\begin{array}{lll}
1 & 1 & 0 \\
\kappa & 1 & 1 \\
1 & 0 & 1
\end{array}\right), M_{11}=\left(\begin{array}{lll}
1 & 1 & 0 \\
1 & 0 & 1 \\
\kappa & 1 & 1
\end{array}\right) \text {. }
\end{aligned}
$$

TABLE 1. Nonzeroth Eigenvalues of the Matrices $w, \kappa=5$.

\begin{tabular}{|c|c|c|}
\hline$[\mathbf{1 0 0}]$ & {$[\mathbf{1 1 0}]$} & {$[\mathbf{1 1 1}]$} \\
\hline \pm 8.8175 & \pm 7.4270 & \pm 6.9176 \\
\hline \pm 2.2361 & \pm 5.4146 & \pm 4.6212 \\
\hline \pm 1.5003 & \pm 2.1830 & \pm 0.9218 \\
\hline & \pm 1.2256 & \pm 0.5774 \\
\hline & \pm 0.8570 & \pm 0.3825 \\
\hline & \pm 0.8259 & \pm 0.3586 \\
\hline & \pm 0.7071 & \\
\hline & \pm 0.5813 & \\
\hline
\end{tabular}

TABLE 2. Nonzeroth Eigenvalues of the Matrices $w, \kappa=1.5$.

\begin{tabular}{|c|c|c|}
\hline$[\mathbf{1 0 0}]$ & {$[\mathbf{1 1 0}]$} & {$[\mathbf{1 1 1}]$} \\
\hline \pm 3.1948 & \pm 3.8230 & \pm 3.9880 \\
\hline \pm 2.2361 & \pm 2.1830 & $\pm \mathbf{1 . 7 7 2 8}$ \\
\hline \pm 1.2422 & \pm 1.6619 & \pm 1.0431 \\
\hline & \pm 1.1114 & \pm 0.5774 \\
\hline & \pm 0.8570 & \pm 0.1377 \\
\hline & \pm 0.7071 & \pm 0.1106 \\
\hline & \pm 0.3364 & \\
\hline & \pm 0.1557 & \\
\hline
\end{tabular}

To proceed further we must know the functions of the local densities of states $\rho_{m}(\omega)$. To find these functions one should specify the parameters for our lattice and the substitutional impurity atom. For qualitative estimations, however, it is possible to involve suitable standard expressions for density functions which in many cases give good approximation.

For qualitative estimations we use the following density functions:

$$
\rho_{m}(\omega)=\lambda_{a m} \rho_{a}(\omega)+\lambda_{o m} \rho_{o}(\omega)
$$

where

$$
\rho_{a}(\omega)=\left(3 \omega / \omega_{a}^{3}\right) \sqrt{\omega_{a}^{2}-\omega^{2}}
$$


is the density function for acoustic phonons and

$$
\rho_{o}(\omega)=\left(8 / \pi\left(\omega_{o 2}-\omega_{o 1}\right)^{2}\right) \sqrt{\left(\omega_{o 2}-\omega\right)\left(\omega-\omega_{o 1}\right)}, \quad \omega_{o 1} \leq \omega \leq \omega_{o 2} .
$$

is the density function for optical phonons, $\lambda_{a m}$ and $\lambda_{o m}$ stand for the corresponding contribution of the phonon bands to the $m$ - th density of state, $\lambda_{a m}+\lambda_{o m}=1, \omega_{a}, \omega_{o 1}$ and $\omega_{o 2}$ are the limiting phonon frequencies for acoustic and optical bands respectively.

Formulas (16) and (17) make a correct account of the Van Hove singularities in the density of states of phonons in the crystal lattice. We should underline, that the two-phonon Green's functions $\tilde{G}_{m}(\omega)$ which are relevant to our process, are less sensitive to details of local dynamics than the one-phonon Green's function $G_{m}(\omega)$.

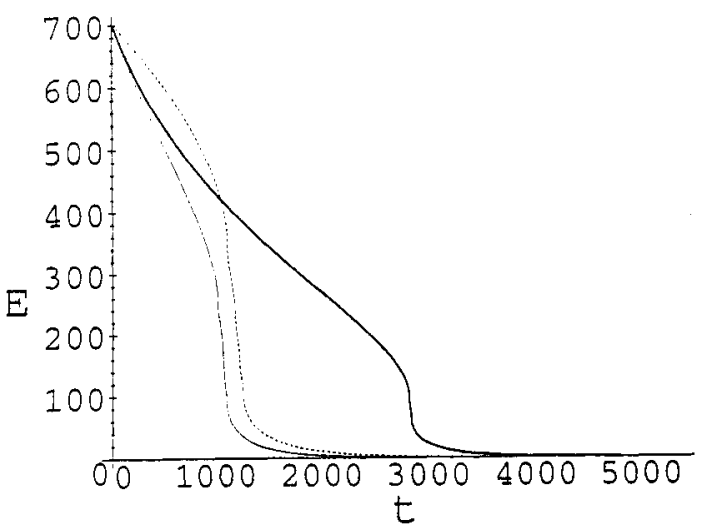

Fig. 2. Relaxation of the energy of the local mode in directions [100] - thick line, [110] - thin line, [111] dotted line. $\kappa=5, E_{l}(0)=1800$.

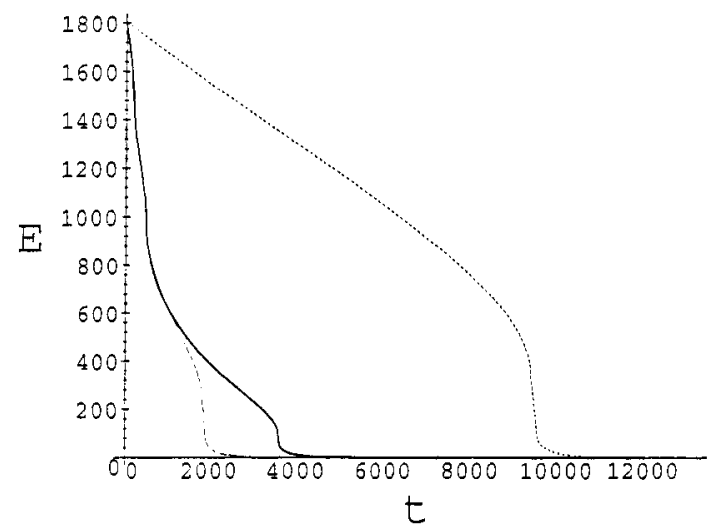

Fig. 4 Relaxation of the energy of the local mode in directions [100] - thick line, [110] - thin line, [111] dotted line. $\kappa=5, E_{l}(0)=1800$.

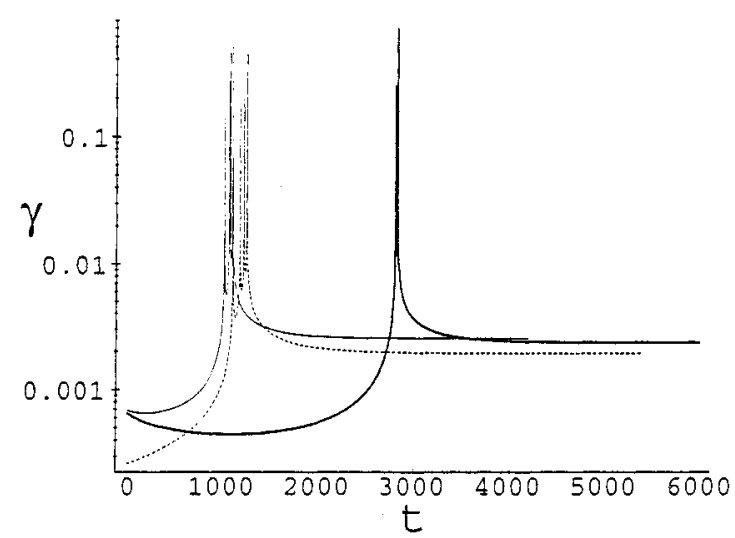

Fig. 3 Rate of the relaxation of the local mode in directions $[100]$ - thick line, [110] - thin line, [111] dotted line. $\kappa=5, E_{l}(0)=1800$.

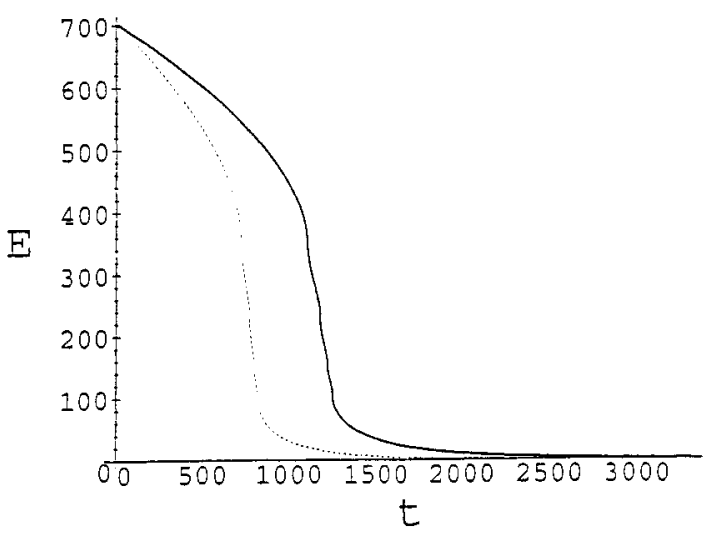

Fig. 5 Relaxation of the energy of the local mode in direction [111], $T=0$ - thick line, $T=0.85$ - thin dotted line. $\kappa=5, E_{l}(0)=700$.

In Figs. 2-5 the critical behaviour of the rate of relaxation of a strongly excited local mode is shown. To simplify calculations we chose a system of units where $\hbar=k=1$. On the plots time is measured in units $\omega_{a}^{-1}$, energy is given in units of vibrational quanta $\omega_{a}, \kappa$ is a parameter of interaction.

The time evolution of the local vibration is illustrated in Figs. 2, 4, 5. The rate of the decay is shown in Fig. 3. As can be seen, the process of relaxation is determined by the set of parameters 
for interaction coefficients and by the initial energy of the excitation of vibration. Depending on the initial conditions, the most stable vibrations can be either in [100] or in [111] direction. This effect of anisotropic relaxation is specific for the mechanism described.

In Figs. 2, 3 the initial energy $\left(E_{l}(0)=700\right)$ and the critical values for directions [110] and [111] are close. Therefore, the vibration in [100] is more stable. The situation is just the opposite in Fig. 4 $\left(E_{l}(0)=1800\right)$, the lifetime of the vibrations in [100] and [110] is much shorter than that of the vibration in [111] direction.

In Fig. 5 a comparative behaviour of relaxation for two temperatures is shown. Generally, for higher temperatures the damping of the local vibration is faster.

Finally we should pay attention to the following features of the described process of generation of phonon bursts by strong local vibration:

1. A remarkable time delay between the excitation of the local vibration moment and the phonon burst, generated by this vibration; this delay may be of hundreds or thousands of vibrational periods, see Fig. 6 - relaxation in direction [111].

2. Quasimonochromatic spectrum of phonons, generated in the burst.

3. Dependence on the direction of vibration. Separate calculations should be carried out for each type of the crystall lattice with a correct account of all possible contributions from vibrations of atoms.

These effects may be used for the experimental observation of the process.

\section{CONCLUSION}

We showed that quantum (and thermal) effects lead to the creation of extra channels in the decay of a strong local vibration (intrinsic or associated with an impurity) with the time-dependence being strongly nonexponetial. At very large energies the rate of the decay is relatively low. It increases during the course of the relaxation, at critical points it gets extremely high values. Then the rate decreases and after that increases again until the energy reaches next critical point. The energy of the local mode drops in a short time at each critical point and a burst of quasimonochromatic phonons is generated. The relaxation rate is higher for higher temperatures, although the values of critical energies do not depend on temperature. We also showed that the law of relaxation depends strongly on the directions of vibration and its behaviour is different for different initial energies. Strong dependence of the relaxation on the directions of vibration obtained for cubic crystals is remarkable, since in the case of the existence of chemical bonds the most stable are vibrations in the direction of the bonds. This effect may have an important value for chemical reactions and for the mechanism of defects formation in solids

It follows from formulae (7) and (8) that the damping rate $\gamma$ of the local mode is strongly enhanced (diverges in our approximation as $\sim\left|t-t_{k}\right|^{-1 / 2}$ ) if the mode energy approaches (at $t=t_{k}$ ) one of the critical energies $E_{k}$. This enhancement of $\gamma$ is associated with the generation of quasimonochromatic phonons. These phonons are emitted in pairs: one phonon with the frequency $\omega_{k}$ and another with the frequency $\left(\omega_{l}-\omega_{k}\right)$. As a result the decay of the strongly excited local mode is highly non-exponential: it has step-wise jumps near critical energies.

At a final stage of decay the relaxation becomes exponential, i.e. the rate of decay is constant. In weak coupling limit the theory presented also gives the constant rate of decay. This is similar to the result given by perturbation theory [20] and to the numerical simulations [6] of classical anharmonic chain.

For experimental observation of the relaxation jumps both, quasimonochromatic spectrum of generated phonons and the time delay between creation of the strong vibration and the jumps can be used. Strong local vibrations can be excited e.g. by light or by high energy particles. Excitation by light takes place e.g. in luminescence centers in ionic crystals with small quantum yield of emission; here, due to the nonradiative transitions, the electronic excitation is abruptly transformed to the local vibrations. High energy particles create in crystals focusons which, in turn, efficiently pass their energy to impurities by excitation of their vibrations. 


\section{ACKNOWLEDGEMENT}

Supported by the Estonian Science Foundation Grant No. 369

\section{References}

1. A.A.Ovchinnikov, N.S.Erihman. Usp. Fiz. Nouk 138, 289 (1982) [Sov. Phys. Usp. 25, 738 (1982)].

2. A.S.Dolgov. FTT 28, 1641 (1986) [Sov. Phys. Solid State 28, 907 (1986)].

3. A.J.Sievers, S.Takeno. Phys. Rev. Lett. 61, 970 (1988).

4. G.S.Zavt, M.Wagner, and A.Lütze. Phys. Rev. E 47, 4108 (1993).

5. J.B.Page. Phys. Rev, B 41, 7835 (1990).

6. V.M.Burlakov, S.A.Kiselev. Zh. Eksp. Teor. Fiz. 99, 1526 (1991).

7. K.W.Sandusky, J.B.Page and K.E.Schmidt. Phys. Rev. B 46, 6161 (1992).

8. V.Hizhnyakov, Proceedings of the XIIth Symposium on the Jahn-Teller Effect [Proc. Estonian Acad. Sci. Phys. Math. 44, 364 (1995)].

9. V.Hizhnyakov. Phys.Rev. B 53, 13981 (1996).

10. V.Hizhnyakov, D.Nevedrov. Proc. Estonian Acad. Sci. Phys. Math. 44, 376 (1995).

11. S.W.Hawking. Nature (London) 243, 30 (1974); Commun. Math. Phys. 43, 199 (1975).

12. N.O.Birel, P.C.Davies. Quantum Fields in Curved Space, Cambridge University Press, Cambridge (1982).

13. A.A.Grib et al. Vacuum Quantum Effects in a Strong Field, Energoizdat, Moscow (1988) (in Russian).

14. W.G.Unruh. Phys. Rev. D 14, 870 (1976).

15. E.Jablonovich. Phys. Rev. Lett. 62, 1742 (1989); in Laser Optics of Condensed Matter, edited by E.Carmier, A.A.Maradudin, and K.K.Rebane. Plenum Press, New York (1991), Vol. 2, p. 325.

16. V.Hizhnyakov. Quantum Optics 4, 277 (1992); Proc. Estonian Acad. Sci. Phys. Math. 41, 211 (1992); in Symposium of the Foundations of Modern Physics 1994; edited by K.V.Laurikainen, Editions Frontiers, Gif-surYvette (1994) p.199.

17. A.A.Maradudin et. al. Theory of Lattice Dynamics in Harmonic Approximation, Academic Press, New York (1963).

18. A.A.Maradudin. Theoretical and Experimental Aspects of the Effects of Point Defects and Disorder of the Vibrations of Crystals, Academic, New York (1966).

19. V.Hizhnyakov. J. Phys. C: Solid State Physics 20, 6073 (1987).

20. P.Klemens. Phys. Rev. 122, 443 (1961).

21. W.Heisenberg, H.Euler. Z.Phys. 98, 714 (1936).

22. E.N.Economou. Green's Functions in Quantum Physics, Springer-Verlag, Berlin (1983). 